\title{
Assessment of Transversus Abdominis Plane Block in Abdominal Surgery by Total Requirement of Diclofenac as a Post-Operative Analgesia Drug
}

\author{
Jigna R Shah ${ }^{1}$, Apoorva Moghe ${ }^{2}$, Maulik Humbal ${ }^{3}$ \\ ${ }^{1}$ Associate Professor, Department of Anaesthesia, GMERS Medical College, Sola Civil Hospital, Sola, Ahmedabad, Gujarat, India, ${ }^{2}$ Second Year Resident, \\ Department of Anaesthesia, GMERS Medical College, Sola Civil Hospital, Sola, Ahmedabad, Gujarat, India, ${ }^{3}$ First Year Resident, Department of \\ Anaesthesia, GMERS Medical College, Sola Civil Hospital, Sola, Ahmedabad, Gujarat, India.
}

\section{Abstract}

Background: Amongst various techniques of TAP block, landmark technique via the triangle of Petit seems to hold considerable promise for patients undergoing surgical procedures involving abdominal wall incisions. The aim of this study is to evaluate transversus abdominis plane [TAP] block in abdominal surgery by total requirement of diclofenac as postoperative analgesia drug. Subjects and Methods: Present study was carried out at Department of Anesthesia, GMERS medical college, Sola, Ahmedabad, Gujarat, India from May 2014 to May 2015. According to formula based nomogram, sample size for proposed study would be: Study group ( $\mathrm{n}=30$ ): patient received TAP block with injection bupivacaine $(0.25 \%) 20 \mathrm{ml}$. Control group $(\mathrm{n}=30)$ : patient not received TAP block and was given injection diclofenac on demand for post-operative analgesia as per institute protocol for routine surgery. Sensory block was assessed by sterile pin prick method in the midaxillary line on both sides of chest. Postoperative pain was assessed by using the visual analogue scale. Results: Majority of the patient were in age group of 30 to 50 in both group. VAS score was significantly higher in control group as compared to the study group at all the time. First dose of rescue analgesia required in study group was at $669.66 \pm 346 \mathrm{~min}$ and in control group was $220.33 \pm 139.24$ min which was statistically significant. Diclofenac requirement in study group was one time in 22 patient and two time in 8 patient which was significantly less as compare to control group in which diclofenac requirement was one time in 2 patient and two time in 5 patient and three time in 23 patient in 24 hour. Conclusion: TAP block is a promising new technique for postoperative pain management in surgery involving the anterior abdominal wall as a part of multimodal analgesia. Further studies are warranted to support this finding before establishing it in routine clinical practice in different type of surgical procedures.

Keywords: Analgesia, Bupivacaine, Diclofenac, Transversus Abdominis Plane.

Corresponding Author: Dr. Apoorva Moghe, Department of Anaesthesia, GMERS Medical College, Sola Civil Hospital, Sola, Ahmedabad, Gujarat, India.

Email: drpiyushpujara@gmail.com

Received: February 2020

Accepted: February 2020

\section{Introduction}

Effective postoperative pain control is an essential component of the care of the surgical patient. Inadequate pain control, apart from being in human, may result in increased morbidity or cost. The advantages of effective postoperative pain management include patient comfort and satisfaction, earlier mobilization, fewer pulmonary and cardiac complications, a reduced risk of deep vein thrombosis, faster recovery with less likelihood of the development of neuropathic pain, and reduced cost of care. Site and invasiveness of surgery as well as type of incision has profound effect on the degree of postoperative pain. The anterior abdominal wall is a significant source of pain after visceral surgery having major adverse effects in many organ systems thus anterior abdominal wall analgesia may assist in improving postoperative outcome. ${ }^{[1,2]}$
In last two decades, laparoscopic visceral surgery has brought great benefits, enabling patients to recover quickly from surgery and allowing them to return to normal activities. Although laparoscopic surgery has been shown to produce less post-operative pain compared to open surgery, there is still the problem of parietal pain arising from the port site and specimen extraction site.

The use of strong pain killing medications such as opioid based drugs which can have a detrimental effect on the postoperative recovery of bowel function and an increased risk of post-operative nausea and vomiting delaying discharge. Amongst options available, afferent neural blockade with local anesthetics seems to be the most effective analgesic technique followed by nonsteroidal anti-inflammatory agents and opioids.

In the last decade, a novel approach to block the abdominal wall neural afferents via the "lumbar triangle of Petit" has been described by Rafi in 2001, ${ }^{[1]}$ known as transversus 
abdominis plane (TAP) block. By introducing the local anesthetics into the transversus abdominis plane (TAP) via the triangle of Petit, it is possible to block the sensory nerves of the anterior abdominal wall before they leave this plane and pierce the musculature to innervate the entire anterior abdominal wall (T7 to L1).

TAP blocks have been described as an effective component of multimodal postoperative analgesia for a wide variety of abdominal procedures including hernia repair, ${ }^{[3]}$ large bowel resection, ${ }^{[20]}$ open / laparoscopic appendectomy, ${ }^{[4-6]}$ cesarean section, ${ }^{[7]}$ total abdominal hysterectomy, laparoscopic cholecystectomy, ${ }^{[13]}$ open prostatectomy, ${ }^{[3]}$ renal transplant surgery, ${ }^{[8]}$ abdominoplasty with / without flank liposuction and iliac crest bone graft. ${ }^{[9-11]}$ Most reports demonstrate the efficacy of TAP blocks by highlighting some combination of reduced postoperative opioid requirement, lower pain scores, and/or reduction in opioid-related side effects.

Amongst various techniques of TAP block, landmark technique via the triangle of Petit seems to hold considerable promise for patients undergoing surgical procedures involving abdominal wall incisions, ${ }^{[12-14]}$ However anatomical studies indicate that the depth and position of the 'triangle of Petit' varies widely in small surface area. Hebbard et al (2007) have subsequently described an ultrasound-guided (USG) approach to the TAP block. Realtime ultrasound provides reliable imaging of the three muscular layers of the anterolateral abdominal wall and assessment of correct needle placement and local anesthetic injection thus potentially increasing the success rate and safety of the TAP block compared to the landmark technique. ${ }^{[15-18]}$ A subcostal TAP block has been described in addition to the posterior TAP block byHebbard in 2008. ${ }^{[19]}$ This can be used to provide analgesia for abdominal surgery extending above the umbilicus. In this variation, the needle entered the skin in an area near the xyphoid and was advanced inferolaterally such that local anaesthetic is delivered to the TAP along thecostal margin.

Børglum et al, ${ }^{[20]}$ recently described an ultrasound-guided, four-point, single-shot technique that combines the posterior and oblique subcostal techniques in an effort to provide wider bilateral analgesic coverage.

Surgeon-Assisted Approaches: While the majority of published literature on TAP blocks is purely from the perspective of anesthesiologists, a growing number of reports have demonstrated that surgeons can help to facilitate these blocks.

Chetwood et al, ${ }^{[21]}$ described a laparoscopic-assisted technique wherein a classic TAP block (based on anatomical landmarks) was performed while the injection area is observed with an intra-abdominal laparoscopic camera. A peritoneal bulge at the area of injection was seen after local anesthetic was delivered within the TAP, and this visual served as the desired endpoint for this technique. Such direct visualization may help to avoid intraperitoneal injection, one of the major potential risks of the TAP block. More recently, a surgical TAP block utilizing a transperitoneal approach was also described. Performed intraoperatively, a blunttipped block needle was advanced from inside the abdominal wall through the parietal peritoneum, then the transversus abdominis muscle, and into the TAP as indicated by a single pop sensation. ${ }^{[22,23]}$ In addition, Araco et al describeda surgical TAP block in which blunt dissection through the external and internal oblique muscles leads to injection of local anesthetic into the TAP under direct visualization.

So, we hypothesized that TAP block could provide safe and reliable block for analgesia to the parietal peritoneum as well as the skin and muscle of the anterior abdominal wall pain after abdominal surgery as a part of multimodal analgesic technique. We selected inguinal hernia meshplasty for study as incision site in lower abdomen which can be blocked by TAP block with landmark technique.

The aim of this study is to evaluate transversus abdominis plane [TAP] block in abdominal surgery by total requirement of diclofenac as postoperative analgesia drug.

\section{Subjects and Methods}

Present study was carried out at Department of Anesthesia, GMERS medical college, Sola, Ahmedabad, Gujarat, India from May 2014 to May 2015. Only indoor patients were selected for elective surgery of inguinal hernioplasty in Department of surgery at GMERS Medical College and Hospital, Sola, Ahmedabad were selected for study. Sample size calculated by review of previous studies. According to these study mean of VAS score in study group was 2 and in control group was 4 and standard deviation was 2 . According to formula based nomogram, sample size for proposed study would be: 30 per group.

Study group $(n=30)=$ patient received TAP block with injection bupivacaine $(0.25 \%) 20 \mathrm{ml}$.

Control group $(n=30)=$ patient not received TAP block and was given injection diclofenac on demand for post-operative analgesia as per institute protocol for routine surgery.

Inclusion Criteria:

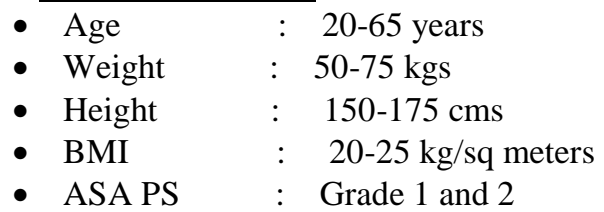

\section{Exclusion Criteria:}

- Patient refusal

- Patient allergic to local anaesthetic

- Infection at puncture site

- Coagulation defects

After obtaining approval from the institutional Ethics Committee and written informed consent, 60 patients of ASA physical status I and II, between 20 to 65 years of age scheduled for elective inguinal hernioplasty were selected for this prospective, randomized and double-blinded controlled study.

As patients were operated for elective inguinal hernioplasty, they were thoroughly examined and assessed preoperatively for any cardiovascular, respiratory or any other systemic illness and were investigated with Complete blood count, blood sugar level, blood urea level, serum creatinine, liver function test, HIV, HBsAg, Urine examination (routine and microscopy), bleeding time and clotting time, Chest X-ray and Electrocardiogram.

All patient were fasted overnight prior to the scheduled day 
of operation. All surgeries were carried out under spinal anaesthesia. On arrival to operation theatre, following insertion of $18 \mathrm{G}$ venous cannula, all patients were received $15 \mathrm{ml} / \mathrm{kg}$ of Ringer's Lactate solution before spinal anaesthesia. Standard monitors like electrocardiography (ECG), Noninvasive Blood Pressure (NIBP) monitoring and Pulse Oximetry were attached and the baseline parameters was recorded.

Lumbar puncture was done at L3-L4 space through a standard $25 \mathrm{G}$ quincke spinal needle in sitting position of patient. Drug $0.5 \%$ hyperbaric bupivacaine $3.5 \mathrm{ml}$ was administered intrathecally as a single dose in all patients. All patients were received oxygen $5 \mathrm{~L} / \mathrm{min}$ via a face mask throughout the procedure after approximating them to the surgical position.

Sensory block was assessed by sterile pin prick method in the midaxillary line on both sides of chest. Immediately after sensory block assessment, motor block was assessed using a modified Bromage scale. The highest dermatomal level of sensory block and recovery times of both sensory and motor block were recorded. Recovery time for the sensory block is defined as two dermatome regression of anaesthesia from the maximal level. Motor block duration is the time to return to grade 0 on the modified Bromage scale. Highest dermatomal level of sensory block after completion of surgery was recorded.

Hypotension will be treated with incremental doses of mephenteramine $3.0 \mathrm{mg}$ i.v. and additional lactated Ringer's solution as appropriate. Bradycardia will be treated with atropine $0.6 \mathrm{mg}$ i.v. After completion of surgery TAP block was performed on the same side of surgery by landmark technique in the study group.

Postoperative pain was assessed by using the visual analogue scale. Patients with a VAS score of 3 or more were received injection diclofenac sodium $75 \mathrm{mg}$ intramuscularly. The time of first request for postoperative analgesia after surgery was recorded as duration of postoperative analgesia. Total dose of inj. diclofenac in $24 \mathrm{hr}$. was recorded. Vital signs, VAS scores, cumulative dose of diclofenac at $30 \mathrm{~min}, 2,4,6,12$, 18 , and $24 \mathrm{hr}$ postoperatively was recorded. Complications of TAP block were noted. All postoperative assessments were made by an investigator blinded to study group.

\section{Statistical Method}

Data was collected,tabulated and then analysed. Data was expressed as mean and standard deviation (SD), median and ranges. Demographic data were analysed using student's ttest.Other measurement like duration of analgesia, total requirement of diclofenac, vas score, vitals were analysed by student t-test. A difference with significant level (p- value< 0.05 ) was considered statistically significant.

\section{Results}

This prospective study was carried out in 60 ASA I and II patients, who underwent inguinal hernioplasty during the year 2014-15.

Table 1: Demographic Data
\begin{tabular}{|l|l|l|l|}
\hline Group & Study Group & Control Group & P Value \\
\hline Age (years)* & $46.43+13.1$ & $50.23 \pm 13.44$ & 0.272 \\
\hline Weight $(\mathrm{kg})^{*}$ & $63 \pm 5.37$ & $61 \pm 5.24$ & 0.1497 \\
\hline Height $(\mathrm{cm})^{*}$ & $167.76 \pm 5.99$ & $167.93 \pm 4.17$ & 0.899 \\
\hline
\end{tabular}

Majority of the patient were in age group of 30 to 50 in both group. Both groups were comparable in terms of age, weight and height. No significant difference was observed between two groups in terms of demographic data.

\section{Table 2: Sensory Level [Thoracic] After Complition of Surgery} or at the Time of Tap Block

\begin{tabular}{l|l|l|}
\hline Case & Control & P Value \\
\hline $\mathrm{T}-7.8 \pm 1.42$ & $\mathrm{~T}-7.6 \pm 1.10$ & 0.5445 \\
\hline $\mathrm{p}>.05$ \\
No significant difference in both groups.
\end{tabular}

Sensory levels [thoracic] are almost same at the end of surgery or at time of the TAP block in both groups.

[Table 3] VAS score was significantly higher in control group as compared to the study group at all the time.

First dose of rescue analgesia required in study group was at $669.66 \pm 346$ min and in control group was $220.33 \pm 139.24$ min which was statistically significant. Total dose of DICLOFENAC consumption in study group was $95 \pm 33.73 \mathrm{mg}$ and in control group it was $202.5 \pm 44.69 \mathrm{mg}$, which showed that DICLOFENAC consumption was significantly decreased in study group.

[Table 4] Tap block with bupivacaine reduced cumulative post-operative DICLOFENAC consumption compared with the control group all the time except at 6 hour.

[Table 5] Heart rate in both group were not significantly different at all the time $(\mathrm{P}>0.05)$ except at the 24 hour where heart rate was significantly high in control group $(\mathrm{P}<0.05)$

[Table 6] We observed that there was no significant difference in MBP in both the group at all the time $(\mathrm{P}>0.05)$ except at 2 hour post-operatively, where MAB is high in control group $(\mathrm{P}<0.05)$.

\section{Table 3: Comparison of Vas Score}

\begin{tabular}{|l|l|l|l|l|l|l|l|l|}
\hline Group & Preoperative & $\mathbf{3 0 m i n}$ & $\mathbf{2 h r}$ & $\mathbf{4 h r}$ & $\mathbf{6 h r}$ & $\mathbf{1 2 h r}$ & $\mathbf{1 8 h \mathbf { h }}$ & $\mathbf{2 4 h \mathbf { h }}$ \\
\hline Study & 0 & $0.4 \pm 0.49$ & $0.9 \pm 0.40$ & $1.36 \pm 0.76$ & $1.9 \pm 1.24$ & $2.23 \pm 1.04$ & $1.66 \pm 0.75$ & $2.2 \pm 0.88$ \\
\hline Control & 0 & $1.03 \pm 0.61$ & $2.3 \pm 1.02$ & $2.9 \pm 1.15$ & $2.46 \pm 0.77$ & $3.8 \pm 0.76$ & $3.13 \pm 0.68$ & $3.66 \pm 0.84$ \\
\hline P value & 0 & $<0.0001$ & $<0.0001$ & $<0.0001$ & $<0.04$ & $<0.0001$ & $<0.0001$ & $<0.0001$ \\
\hline P* $<0.05$ &
\end{tabular}

Table 4: Cumulative Consumption of Diclofenac

\begin{tabular}{|l|l|l|l|l|l|l|l|l|}
\hline Group & Preoperative & $\mathbf{3 0 m i n}$ & $\mathbf{2 h r}$ & $\mathbf{4 h r}$ & $\mathbf{6 h r}$ & $\mathbf{1 2 h r}$ & $\mathbf{1 8 h r}$ & $\mathbf{2 4 h \mathbf { h }}$ \\
\hline Study & 0 & 0 & 0 & $7.5 \pm 22.88$ & $22.5 \pm 34.95$ & $30 \pm 37.37$ & $7.5 \pm 22.88$ & $27.5 \pm 36.75$ \\
\hline Control & 0 & 0 & $22.05 \pm 34.95$ & $35 \pm 38.05$ & $10 \pm 25.93$ & $62.5 \pm 28.42$ & $15 \pm 30.51$ \\
\hline P value & 0 & 0 & 0 & 0.0014 & 0.1216 & 0.0004 & 0.2862 \\
\hline
\end{tabular}


Table 5: Comparisons of Mean Heart Rate

\begin{tabular}{|l|l|l|l|l|l|l|l|l|}
\hline Group & Preoperative & $\mathbf{3 0 m i n}$ & $\mathbf{2 h r}$ & $\mathbf{4 h r}$ & $\mathbf{6 h r}$ & $\mathbf{1 2 h r}$ & $\mathbf{1 8 h r}$ \\
\hline Study & $76.2 \pm 6.81$ & $73.2 \pm 6.94$ & $73.66 \pm 6.78$ & $75.63 \pm 6.59$ & $76.53 \pm 6.59$ & $77.46 \pm 8.03$ & $76.66 \pm 6.30$ & $77.13 \pm 6.11$ \\
\hline Control & $76.76 \pm 7.99$ & $72.26 \pm 7.40$ & $75.03 \pm 8.55$ & $77.1 \pm 8.45$ & $76.7 \pm 8.57$ & $77.56 \pm 15.69$ & $78.6 \pm 8.10$ & $81 \pm 7.87$ \\
\hline P value & 0.7712 & 0.6137 & 0.4945 & 0.4557 & 0.9317 & 0.9754 & 0.305 \\
\hline
\end{tabular}

Table 6: Comparison of Mean Arterial Blood Pressure

Table 6: Comparison of Mean Arterial Blood Pressure
\begin{tabular}{|l|l|l|l|l|l|l|l|l|}
\hline Group & Preoperative & $\mathbf{3 0 m i n}$ & $\mathbf{2 h r}$ & $\mathbf{4 h r}$ & $\mathbf{6 h r}$ & $\mathbf{1 2 h r}$ & $\mathbf{1 8 h r}$ \\
\hline Study & $95.24 \pm 9.44$ & $88.82 \pm 6.21$ & $90.6 \pm 5.11$ & $93.24 \pm 5.91$ & $94.51 \pm 5.88$ & $96.44 \pm 6.26$ & $94.15 \pm 5.51$ & $96.95 \pm 4.38$ \\
\hline Control & $97.48 \pm 9.40$ & $91.33 \pm 5.82$ & $94.46 \pm 5.50$ & $95.66 \pm 5.92$ & $95.26 \pm 5.34$ & $98.46 \pm 5.64$ & $96.42 \pm 4.51$ & $99.44 \pm 5.61$ \\
\hline P value & 0.3609 & 0.1117 & 0.0066 & 0.1185 & 0.607 & 0.1944 & 0.0863 & 0.0606 \\
\hline
\end{tabular}

\begin{tabular}{l|l|l|l|l|l|l|l|l|}
\hline Table 7: Comparison of Respiratory Rate \\
\hline Group & Preoperative & $\mathbf{3 0 m i n}$ & $\mathbf{2 h r}$ & $\mathbf{4 h r}$ & $\mathbf{6 h r}$ & $\mathbf{1 2 h r}$ & $\mathbf{1 8 h r}$ \\
\hline Study & $14.4 \pm 0.72$ & $14.3 \pm 0.74$ & $14.56 \pm 0.89$ & 14.86 v 1.07 & $15.13 \pm 0.93$ & $15.13 \pm 0.97$ & $14.46 \pm 0.75$ & $14.5 \pm 0.77$ \\
\hline Control & $14.5 \pm 0.77$ & $14.46 \pm 0.68$ & $14.9 \pm 0.84$ & $15.33 \pm 0.80$ & $14.93 \pm 0.63$ & $15.73 \pm 0.44$ & $14.9 \pm 0.75$ & $15.5 \pm 0.62$ \\
\hline P value & 0.6053 & 0.3868 & 0.1335 & 0.0593 & 0.3341 & 0.0037 & 0.0268 & 0.0001 \\
\hline
\end{tabular}

Table 8: Number of Patient Who Required Diclofenac in 24 Hour

\begin{tabular}{|c|c|c|}
\hline & Study group & Control group \\
\hline No. of patients who did not need any rescue analgesic once in 24 hours & 0 & 0 \\
\hline No. of patients who received rescue analgesic once in 24 hours & 22 & 2 \\
\hline No. of patients who received rescue analgesic twice in 24 hours & 8 & 5 \\
\hline No. of patients who received rescue analgesic thrice in 24 hours & 0 & 23 \\
\hline
\end{tabular}

[Table 7] Respiration rate is high in control group at 12 hours, 18 hours and 24 hour $(\mathrm{P}<0.05)$ only otherwise there is no difference all the time $(\mathrm{P}>0.05)$.

Diclofenac requirement in study group was one time in 22 patient and two time in 8 patient which was significantly less as compare to control group in which diclofenac requirement was one time in 2 patient and two time in 5 patient and three time in 23 patient in 24 hour. There were no any complications related to the TAP block technique in both the groups.

\section{Discussion}

Inadequately controlled post-operative pain has undesirable physiological and psychological consequences such as morbidity, delayed recovery and patient dissatisfaction. Safety and morbidity issues are therefore of utmost importance and should be constantly re-evaluated and optimized. Thus, the development of safe and well-tolerated analgesic techniques that provide optimal postoperative pain relief is of utmost importance.

Following inguinal hernia surgery, moderate or severe pain may cause increase the duration of hospital stay, unexpected rehospitalization, delay in returning to normal activities and increase in associated costs. ${ }^{[24,25]}$ Callesen et al. ${ }^{[25]}$ found out moderate or severe pain scores in $60 \%$ of cases in the first day of herniorrhaphy and in $33 \%$ of cases in the 6th day of surgery. Moreover, it was suggested that insufficient postoperative pain management following herniorrhaphy might be a risk factor for the development of chronic pain. ${ }^{[26]}$ Various methods and medications are used in postoperative pain management. The most common approach to postoperative pain relief is multimodalusing NSAIDs, opioids and local infiltration of local anesthetic. Local infiltration does not relieve deep muscular pain and NSAID are nephrotoxic. Peripheral nerve blocks with local anesthetics are a method that may be used in inguinal hernia surgeries for pain management. Iliohypogastric (IH) and ilioinguinal (II) nerve with lower intercostal nerve (T-11 and T-12) block are used for this purpose. Hence we thought of an alternative technique in the form of unilateral TAP block with $0.25 \%$ of Bupivacaine at the end of surgery to evaluate whether it has any opioid sparing effect.

In this study we decided to give TAP block by landmark technique after completion of surgery to prolong the analgesic effect as effect of spinal anaesthesia wear off in 3 to 4 hours. The results of our study showed that patients who received TAP block with local anesthetic had 10 to 12 hour duration of analgesia, significantly less pain up to $24 \mathrm{hrs}$., prolonged time to first dose of rescue analgesic and significant reduction in diclofenac consumption in $24 \mathrm{hrs}$ and the subsequent dose of diclofenac were required at longer time intervalas compared to control group. VAS score was low in study group as compared to control group throughout 24 hours. This was similar to the study performed by $\mathrm{Mc}$ Donnell et al, ${ }^{[12]}$ in caesarean section delivery where VAS at rest and on movement was significantly low till 12 hours in patients who received TAP block with local anesthetic. Study performed by Niraj et al, ${ }^{[4]}$ in open appendicectomy and Neerja Bharti et al, ${ }^{[2]}$ in colorectalsurgery also showed similar results.

Time to first dose of rescue analgesia was statistically significantly prolonged in study group (669.66 \pm 346 minutes) compared to the control group $(220.33 \pm 139.24$ minutes $)$. In studies performed by Mc Donnell et al, ${ }^{[12]}$ and D. Belavy et al, ${ }^{[20]}$ in caesarean section, the time to first analgesic demand was 3-4 hours after completion of surgical procedure which was less compared to our study. This difference may be attributed to TAP block given before surgery whereas we have given TAP block at the end of surgery. Also one time bolus injections of local anesthetics can provide narcoticlimiting pain relief for 4-8 h after operation however it was about 12 hrs in our study. Other studies also demonstrated that a single shot TAP technique can produce effective 
analgesia for 36-48 hrs attributing it to relatively poor vascularised and therefore slow drug clearance. ${ }^{[3]}$

One important measure of analgesic efficacy in our study was requirement of diclofenac during first $24 \mathrm{hrs}$. The 24 hours consumption of total diclofenac was $53 \%$ less in study group as compared to control group. The cumulative diclofenac consumption at 2, 4, 12, 18, 24 hours was also significantly less in study group compared to the control group. Our study results are comparable with A.A. Dawlatly et $\mathrm{al}^{[15]}$ who showed $55 \%$ decrease in opioid requirement after USG guided TAP block in laparoscopic cholecystectomy. G. Niraj et al4showed $45 \%$ decrease in opioid requirement with USG guided TAP block in open appendicectomy $\mathrm{Mc}$ Donnell et al, ${ }^{[12]}$ also showed $47 \%$ decrease in opioid requirement with TAP block after total abdominal hysterectomy Jumana $M$ Baaj et al, ${ }^{[27]}$ also showed total consumption of morphine was reduced more than $60 \%$ after the TAP block in caesarean delivery under spinal anaesthesia.

Subcostal TAP catheter was used by Ozelsel et al, ${ }^{[28]}$ in patients undergoing elective right hepatectomy and they reported significant opioid sparing in these patients. They used intermittent boluses of $40 \mathrm{ml} 0.2 \%$ ropivacaine every 6 hours with PCA morphine, while Harish et al, ${ }^{[29]}$ used the low-dose infusion via unilateral catheter in open nephrectomy patients and reported that there was no need of opioids for the next 3 days.

In unilateral surgery, it can be given unilaterally. Compared to epidural block, there is absence of sympathetic or motor deficit and potential damage to the spinal cord. Kadam and Field have evaluated the role of ultrasound-guided continuous TAP block for abdominal surgery and observed reduction in postoperative pain scores and fentanyl requirement. ${ }^{[30]}$

Epidural catheter for postoperative analgesia is still gold standard but TAP block can be given for postoperative analgesia when epidural catheter is contraindicated in high risk patient or failure.

Clinical studies involving TAP block so far have shown analgesic efficacy but none of them have investigated the distribution of the TAP block by sensory testing. There has been controversy regarding spread of L/A when injected in TAP. An anatomical study in cadavers with dye injectionfrom ultrasound-guided TAP block by Tran et al, ${ }^{[18]}$ showed that injection cephalad to the iliac crest blocks T10L1 nerve roots. These finding is consistent with Shibata et al who assessed the sensory block by pinprick in 26 patients after ultrasound-guided TAP block for laparoscopic gynaecological surgery. Both advocated that the technique may be limited to use in lower abdominal surgery. In contrast, a study by McDonnell and colleagues reported a sensory block from $\mathrm{T} 7$ to $\mathrm{L} 1$ in three volunteers when a landmark technique was used to access the TAP through the lumbar triangle. ${ }^{[12]}$ This may be related to different approach used in this study. We also did not assess sensory block as it was performed at the end of surgery in patients who received spinal anaesthesia.

Although initial results of TAP block are encouraging, its complications are not well described in the literature but few case reports of complication including like bowel puncture, nerve injury and puncture of the liver. There is one report of liver injury with landmark technique in a short stature woman whose liver was enlarged. We did not observe any of these complications by landmark technique.

Another important concern is L/toxicity particularly when bilateral blocks are performed as administration of local anesthetic between fascia layers is associated with fast absorption kinetics. One study showed that TAP block has the potential to cause systemic toxicity if local anesthetic spills over into the adjacent muscles. We did not encounter this complication as surgical procedure selected required unilateral block and we did not cross the toxic dose of bupivacaine.

Haemodynamic parameters like HR, MAP and RR remained comparable in both the group at all the times. TAP block with bupivacaine showed stable hemodynamic in postoperative period because of good postoperative pain control in early period. Opioid related side effects like sedation, nausea and vomiting are related to the dose of opioids which were absentin our study as we did not use opioid.

From this study, it seems that TAP block holds considerable promise for patients undergoing surgical procedures involving abdominal wall incisions as it provide analgesia to parietal peritoneum as well as skin and muscle of the anterior wall abdomen. It reduces pain (vas score), requirement of rescue analgesia and their side effect and provide better satisfaction to patient.

Advantage of TAP block by landmark technique over USG guided are easy to perform, cost effective, less time required for preparation to give TAP block, can be used at peripheral health center, can be used in emergency surgery when USG not available, can be given by anesthesiologist without expertise in USG guided block.

However further large well controlled studies are required in terms of its safety, optimal dose and volume of local anesthetic, single shot verses catheter, intermittent verses continuous catheter infusion, type of local anesthetic, use of adjuvant, comparison of efficacy of TAP block with other technique like epidural catheter and local infiltration, use in patient with coagulation profile abnormality before it can be implemented in routine clinical practice.

\section{Conclusion}

TAP block is a promising new technique for postoperative pain management in surgery involving the anterior abdominal wall as a part of multimodal analgesia. Further studies are warranted to support these findings before establishing it in routine clinical practice in different type of surgical procedures.

\section{References}

1. Rafi A. Abdominal field block: a new approach via the lumbar triangle. Anaesthesia 2001; 56: 1024-26

2. McDonnell J, O' Donnell B, Tuite D, Farrell T, Power C. The regional abdominal field infiltration (R.A.F.I.) technique: computerized tomographic and anatomical identification of a novel approach to the transverses abdominis neuro-vascular fascial plane Anesthesiology 2004; 101: A899.

3. Aveline $\mathrm{C}$, Le Hetet $\mathrm{H}$ et al Comparison between ultrasound-guided transverses abdominis plane and conventional ilioinguinal / 
iliohypogastric nerve blocks for day-case open inguinal hernia repair. Br J Anaesth 2011; 106 (3): 380-6.

4. Niraj G, Searle A, Mathews M, Misra V, Baban M, Kiani S, Wong M. Analgesic efficacy of ultrasound-guided transverses abdominis plane block in patients undergoing open appendicectomy. $\mathrm{Br} \mathrm{J}$ Anaesth 2009; 103:601-5

5. Sandeman DJ, Bennett $M$ et al. Ultrasound-guided transversus abdominis plane blocks for laparoscopic appendicectomy in children: a prospective randomized trial. Br J Anaesth 2011; 106 (6): 882-6.

6. Block Provides Effective Analgesia After Appendectomy in Children: A Randomized Controlled Trial. Anesth Analg 2010;111:998-1003.

7. Carney J, McDonnell JG, et al The Transversus Abdominis Plane Block Provides Effective Postoperative Analgesia in Patients Undergoing Total Abdominal Hysterectomy. Anesth Analg 2008;107:2056 -60.

8. Mukhtar K, Khattak I. Transversus abdominis plane block for renal transplant recipients. Br J Anaesth 2010; 104(5): 663-664.

9. Araco A, Pooney J, Araco F, Gravante G. Transversus abdominis plane block reduces the analgesic requirements after abdominoplasty with flank liposuction. Ann Plastic Surg 2010; 65(4) : 385-388.

10. Sforza M, Andjelkov K, Zaccheddu R, Nagi H, Colic M. Transversus abdominis plane block anesthesia in abdominoplasties. Plastic Reconstructive Surg 2011;128(2): 529-535.

11. Chiono J, Bernard N, Bringuier S. The ultrasoundguided transversus abdominis plane block for anterior iliac crest bone graft postoperative pain relief: a prospective descriptive study. Reg Anesth Pain Medicine 2010; 35(6): :520-524.

12. McDonnell J, O’Donnell, Brian M, Curley G, Heffernan A, Power C, Laffey J. The Analgesic Efficacy of Transversus Abdominis Plane Block After Abdominal Surgery: A Prospective Randomized Controlled Trial. Anaesth Analg 2007; 104: 193-7.

13. McDonnell J, Curley G, Carney J, Benton A, Costello J, Maharaj C, Laffey J. The Analgesic Efficacy of Transversus Abdominis Plane Block after Cesarean Delivery: A Randomized Controlled Trial. Anesth Analg 2008; 106: 186-91

14. Bachmann A, Giannini O, Wolff T, et al. Retroperitoneoscopic living donor nephrectomy: A comparison with the open approach in respect of early post-operative pain management. Transplantation proceedings 2005; 37:609-612.

15. El-Dawlatly A, Turkistani A et al Ultrasound-guided transversus abdominis plane block: description of a new technique and comparison with conventional systemic analgesia during laparoscopic cholecystectomy. Br J Anaesth 2009; 102: 763-767.

16. Hebbard P. Website: www.heartweb.com.au/download TAP block. pdf

17. Hebbard P, Fujiwara Y, Shibata Y, Royse C. Ultrasound-guided transversus abdominis plane (TAP) block. Anaesth Int Care 2007; 35 616-8

18. Tran TM, Ivanusic JJ, Hebbard P, et al. Determination of spread of injectate after ultrasound-guided transversus abdominis plane block: a cadaveric study. Br J Anaesth 2009;102(1):123.

19. Hebbard P. Subcostal transversus abdominis plane [TAP] block under ultrasound guidance. Anaesth Analg 2008; 106(2) : 675-8.

20. Belavy D, Cowlishaw PJ, Howes M, Phillips F. Ultrasoundguided transversus abdominis plane block for analgesia after caesarean delivery. Br J Anaesth 2009;103:726-30.

21. Ghassan E. Kanazi, Marie T. Aouad, et al The Analgesic Efficacy of Subarachnoid Morphine in Comparison with Ultrasound-Guided Transversus Abdominis Plane Block After Cesarean Delivery: A Randomized Controlled Trial. Anesth Analg 2010;111:475-81.

22. Bharti N, Kumar P, Bala I, Gupta V. The efficacy of a novel approach to transversus abdominis plane block for postoperative analgesia after colorectal surgery. Anesth Analg 2011; 112(6): 1504-1508.

23. Owen DJ, Harrod I, Ford J, Luckas M, Gudimetla V. The surgical transversus abdominis plane block-a novel approach for performing an established technique. Br J Obs Gynaecol 2011; 118 (1): 24-27.

24. Willschke $\mathrm{H}$, Marhofer P, Bösenberg A, et al. Ultrasonographyfor ilioinguinal/iliohypogastric nerve blocks in children. Br JAnaesth. 2005; 95: 226-30

25. Callesen T, Bech K, Nielsen R. Pain after groin hernia repair. BrJ Surg. 1998; 85: 1412-4

26. Joshi GP. Multimodal analgesia techniques and postoperative rehabilitation. Anesthesiol Clin North Am. 2005; 23:185-202.

27. Baaj JM, Alsatli RA et al. efficacy of ultrasound-guided transversus abdominis plane (tap) block for postcesarean section delivery analgesia. M.E.J. Anesth 2010; 20 (6): 821-826.

28. Ozelsel T. Subcostal approach for transversus abdominis plane block under direct surgical placement. Reg Anaesth Pain Med. 2009; 32: 90.

29. Harish R. Low-dose infusion with 'surgical transverse abdominis plane (TAP) block' in open nephrectomy. Br J Anaesth. 2009; 102: 889-90

30. Kadam RV, Field JB. Ultrasound guided Continuous Transverse Abdominis plane block for abdominal surgery. J Anaesth Clin Pharmacol. 2011; 27:37-44.

Copyright: (C) the author(s), 2020. It is an open-access article distributed under the terms of the Creative Commons Attribution License (CC BY 4.0), which permits authors to retain ownership of the copyright for their content, and allow anyone to download, reuse, reprint, modify, distribute and/or copy the content as long as the original authors and source are cited.

How to cite this article: Shah JR, Moghe A, Humbal M. Assessment of Transversus Abdominis Plane Block in Abdominal Surgery by Total Requirement of Diclofenac as a Post-Operative Analgesia Drug. Acad. Anesthesiol. Int. 2020;5(1):41-46.

DOI: dx.doi.org/10.21276/aan.2020.5.1.8

Source of Support: Nil, Conflict of Interest: None declared. 